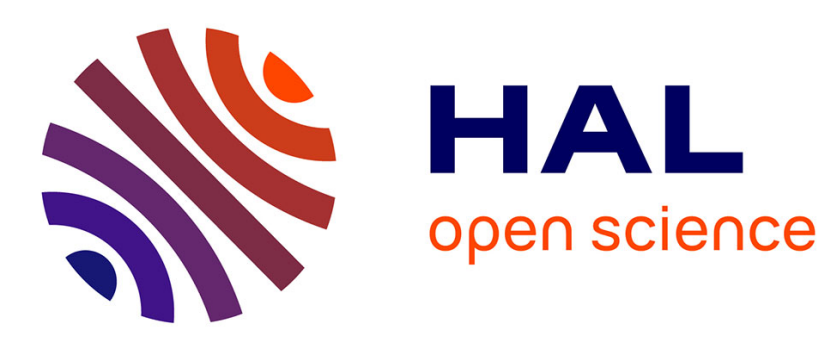

\title{
Stabilizing Integral Delay Dynamics and Hyperbolic Systems using a Fredholm Transformation
}

Jeanne Redaud, Jean Auriol, Silviu-Iulian Niculescu

\section{To cite this version:}

Jeanne Redaud, Jean Auriol, Silviu-Iulian Niculescu. Stabilizing Integral Delay Dynamics and Hyperbolic Systems using a Fredholm Transformation. 60th Conference on Decision and Control (CDC2021), Dec 2021, Austin, Texas, United States. hal-03357334

\section{HAL Id: hal-03357334 https://hal.science/hal-03357334}

Submitted on 28 Sep 2021

HAL is a multi-disciplinary open access archive for the deposit and dissemination of scientific research documents, whether they are published or not. The documents may come from teaching and research institutions in France or abroad, or from public or private research centers.
L'archive ouverte pluridisciplinaire HAL, est destinée au dépôt et à la diffusion de documents scientifiques de niveau recherche, publiés ou non, émanant des établissements d'enseignement et de recherche français ou étrangers, des laboratoires publics ou privés. 


\title{
Stabilizing Integral Delay Dynamics and Hyperbolic Systems using a Fredholm Transformation
}

\author{
Jeanne Redaud $^{1}$, Jean Auriol ${ }^{1}$, and Silviu-Iulian Niculescu ${ }^{1}$
}

\begin{abstract}
In this paper, we design a stabilizing statefeedback control law for a system represented by a general class of integral delay equations. Under an appropriate spectral controllability assumption, an implementable control law is proposed. The approach is constructive and makes use of the well-known backstepping methodology. Due to the integral terms present in the original system, the proposed problem requires a Fredholm transform, which is not always invertible. The invertibility of this transformation is proved using an operator formulation. In particular, we show that this invertibility property is a consequence of spectral controllability. The existence of the kernels defining the Fredholm transform is proved similarly by showing that they satisfy an invertible integral equation. Some test case simulations complete the paper.
\end{abstract}

\section{INTRODUCTION}

In this paper, we design a state-feedback control law that stabilizes a general type of Integral Delay Equations (IDE). They can represent a wide variety of systems. Indeed, numerous engineering or biological models involve transport, communication, or measurement delays [20]. In particular, such delays naturally appear when considering networks of interconnected subsystems. More specifically, IDEs naturally arise when modeling propagation of electric pulses in the heart dynamics [10], for instance, population dynamics or biomedical systems, as epidemics, using transmission delays in their representation [7].

When analyzing such systems, one of the difficulties to consider is that they combine pointwise and distributed delays. In most cases, such delays may induce instabilities of the closed-loop system or bad performances of outputfeedback control laws. Various problems arise in implementing control laws that include distributed delays [21]. Initially, various ideas from complex analysis have been used to find necessary and sufficient stabilizability and controllability conditions [13], [5]. However, these conditions guarantee the existence of a stabilizing state feedback control, but are in most of the cases not operational.

It has been shown in recent contributions that a large class of hyperbolic systems can be rewritten as difference equations with distributed delays, for instance in [2], using a backstepping approach [16]. Delay terms are implied by the transport phenomena inherent to hyperbolic systems and to in-domain couplings (convection terms, for instance). Interestingly, when considering under-actuated systems of hyperbolic equations [1], such distributed delays also appear in the actuation, thus emphasizing the existing links between hyperbolic systems and IDEs. In this context, it is interesting to explore the cases further when specific methods developed for hyperbolic systems can be applied to stabilize IDEs.

${ }^{1}$ Jeanne Redaud, Jean Auriol, and Silviu-Iulian Niculescu are with Université Paris-Saclay, CNRS, Inria, CentraleSupélec, Laboratoire des Signaux et Systèmes, 91190, Gif-sur-Yvette, France. Corresponding author: jeanne.redaudacentralesupelec.fr
In this paper, we propose a constructive approach to design an implementable control law that stabilizes the considered class of IDEs. More precisely, the contribution of the paper is three-fold. First, unlike classical approaches, we use a Partial Differential Equation (PDE) control-oriented framework to stabilize the considered class of IDEs. Then, the proposed approach requires the introduction of an appropriate Fredholm integral transformation. To the best of the authors' knowledge, the existence of this transformation represents a novelty in the literature and corresponds to a generalization of the constructive backstepping technique. Finally, it is worth mentioning that our methodology can be extended to underactuated hyperbolic systems.

The proposed approach can be resumed as follows. We first define a comparison system ${ }^{1}$ [20] described by a set of hyperbolic PDEs including control inputs. We will show that its closed-loop stability properties imply those of the original IDE system. Next, the use of the backstepping methodology allows mapping this hyperbolic system to a stable target system and the design of a stabilizing control law. Unlike traditional approaches [15], the specific structure of the original system requires the use of a Fredholm integral transform. Contrary to Volterra transformations, the existence and invertibility of such a transformation are not guaranteed. Several results in the literature deal with the invertibility of Fredholm transforms when kernels have a specific structure [9], [6]. As these conditions are not fulfilled here, we use an operator framework, as suggested by [8]. More precisely, we show that the well-posedness and invertiblity of our transform represent a consequence of a natural spectral controllability assumption.

Notations: Inspired by [14], for any fixed $\tau>0$, we denote $D_{\tau}=H^{1}([-\tau, 0], \mathbb{R})$ the Banach space of $H^{1}$ real-valued functions mapping the interval $[-\tau, 0]$ into $\mathbb{R}$. For a function $\phi:[-\tau, \infty) \mapsto \mathbb{R}$, its partial trajectory $\phi_{[t]} \in D_{\tau}$ is defined by $\phi_{[t]}(\theta)=\phi(t+\theta),-\tau \leq \theta \leq 0$. The associated $L^{2}$ norm is given by $\left\|\phi_{[t]}\right\|_{L^{2}}=\left(\int_{-\tau}^{0} \phi(t+\theta)^{2} d \theta\right)^{\frac{1}{2}}$. We denote $\mathcal{S}=[0,1]^{2}, \mathcal{T}^{-}=\left\{(x, y) \in[0,1]^{2}, x \geq y\right\}$ and $\mathcal{T}^{+}=$ $\left\{(x, y) \in[0,1]^{2}, x \leq y\right\}$.

\section{PROBLEM UNDER CONSIDERATION}

\section{A. Time-delay formulation}

Consider two known positive delays $\tau_{0}>0$ and $\tau_{1}>0$ and let us define the bounded linear operators $\mathcal{L}_{i}: D_{\tau_{i}} \rightarrow \mathbb{R}$, $i \in\{0,1\}$, by

$$
\mathcal{L}_{i}: \phi_{[t]} \mapsto a_{i} \phi_{[t]}\left(-\tau_{i}\right)+\int_{0}^{\tau_{i}} f_{i}(\nu) \phi_{[t]}(-\nu) d \nu,
$$

\footnotetext{
${ }^{1}$ see, e.g., [20] and the references therein for some discussions on delay systems
} 
with $a_{0} \in(-1,1), a_{1} \in \mathbb{R} \backslash\{0\}$ and $f_{0}, f_{1}$ two piecewise continuous functions. Consider the following time-delay equation defined for all $t \geq 0$ by

$$
\phi_{[t]}=\mathcal{L}_{0} \phi_{[t]}+\mathcal{L}_{1} V_{[t]},
$$

with initial data given by $\phi_{0}=\phi^{0} \in D_{\tau_{0}}$. The function $V \in$ $D_{\tau_{1}}$ corresponds to the actuation, with $V(t)=0, \forall t \leq 0$.

A function $\phi:\left[-\tau_{0}, \infty\right) \rightarrow \mathbb{R}$ is called a solution of the initial value problem (2) if $\phi_{0}=\phi^{0}$, and if (2) is satisfied for $t \geq 0$. Since the coefficient $\left|a_{0}\right|$ is strictly inferior to one, the open-loop system only has a finite number of unstable roots [14]. More precisely, the principal part of the system has to be exponentially stable. If such a condition is not fulfilled, then it is impossible to delayrobustly stabilize the system (2) [18]. Due to the distributed delay term $\left(\int_{0}^{\tau_{0}} f_{0} \phi(t-\nu) d \nu\right)$, the open-loop system may be unstable. The objective of this paper is to design a control law $V(t)$ that exponentially stabilizes the system in the sense of the $D_{\tau_{0}}$-norm. More precisely, we want to design a control law such that $\exists \nu>0, C_{0}>0, \forall \phi^{0} \in D_{\tau_{0}}$, all solutions of the closed-loop (2) satisfy $\left\|\phi_{[t]}\right\|_{L^{2}\left(\left[-\tau_{1}, 0\right]\right)} \leq$ $C_{0} \mathrm{e}^{-\nu t}\left\|\phi^{0}\right\|_{L^{2}\left(\left[-\tau_{1}, 0\right]\right)}$.

Note that the actuation in (2) appears through both pointwise and distributed delay terms. It has been seldom studied in the literature [4], [23], and is a major difference compared to existing results. However, we assume here that there is (at least) a pointwise delay on the actuation since $a_{1} \neq 0$. Obviously, the difficulties to stabilize equation (2) are related to the simultaneous presence of a distributed-delay term on the actuation and on the state. Let us formally take the Laplace transform of equation (2) (zero initial condition). We have $F_{0}(s) \phi(s)=F_{1}(s) V(s)$, where the holomorphic function $F_{0}$ and $F_{1}$ are defined by

$$
\begin{aligned}
& F_{0}(s)=1-a_{0} \mathrm{e}^{-\tau_{0} s}-\int_{0}^{\tau_{0}} f_{0}(\nu) \mathrm{e}^{-\nu s} d \nu, \\
& F_{1}(s)=a_{1} \mathrm{e}^{-\tau_{1} s}+\int_{0}^{\tau_{1}} f_{1}(\nu) \mathrm{e}^{-\nu s} d \nu .
\end{aligned}
$$

To guarantee the possibility to stabilize system (2), we make the following (spectral) controllability assumption

Assumption 1: Spectral controllability [19], [22]

For all $s \in \mathbb{C}, \operatorname{rank}\left[F_{0}(s), F_{1}(s)\right]=1$.

In other words, this assumption says that $F_{0}$ and $F_{1}$ cannot simultaneously vanish.

\section{B. Control strategy}

The control strategy we propose is as follows:

- first, we rewrite the integral delay equation (2) as a hyperbolic system, and prove that this hyperbolic system shares the same stability properties;

- second, using an invertible Fredholm integral transform, we map this hyperbolic system to a stable target system. The invertibility of the backstepping transformation is shown using an operator framework;

- next, to show the existence of the backstepping transformation, we rewrite the kernel equations as integral equations. Again, the existence of a solution to these Fredholm integral equations is shown using an operator framework;

- finally, this leads to a stabilizing state-feedback control law for the original system.

\section{PDE FORMULATION}

In this section, we introduce a simple hyperbolic system that will be useful to conclude to the stability of the original system (2). Indeed, its stability properties imply appropriate stability properties for the "original" time-delay system (2). In other words, this system may be seen as a comparison system for (2). Its well-posedness is shown using an operator formulation. Finally, we conclude this section by giving some general results on Fredholm transformations.

\section{A. A new hyperbolic system}

In this section, we introduce a new hyperbolic system. It corresponds to two conservation laws, with integral terms acting at one boundary.

1) System equations: Define $\lambda=\frac{1}{\tau_{0}}, \mu=\frac{1}{\tau_{1}}, \rho=a_{0}$ and the piecewise continuous functions $N_{u}, N_{v}$ by $N_{u}(x)=$ $\frac{1}{\lambda} f_{0}\left(\frac{1}{\lambda} x\right)$ and $N_{v}(x)=\frac{1}{\mu a_{1}} f_{1}\left(\frac{1}{\mu}(1-x)\right)$. Consider the following hyperbolic system of state $(u(t, x), v(t, x))^{T}$ with $(t, x) \in \mathbb{R}^{+*} \times[0,1]$

$$
\begin{aligned}
& \partial_{t} u(t, x)+\lambda \partial_{x} u(t, x)=0, \\
& \partial_{t} v(t, x)-\mu \partial_{x} v(t, x)=0,
\end{aligned}
$$

with the boundary conditions

$$
\begin{aligned}
u(t, 0)= & v(t, 0)+\rho u(t, 1) \\
& +\int_{0}^{1} N_{u}(y) u(t, y)+N_{v}(y) v(t, y) d y, \\
v(t, 1)= & \bar{V}(t)=a_{1} V(t) .
\end{aligned}
$$

The initial conditions $u_{0}$ and $v_{0}$ are defined for all $x \in[0,1]$ by $u_{0}(x)=u(0, x)=\phi^{0}\left(-\frac{x}{\lambda}\right)$ and $v_{0}(x)=v(0, x)=0$. They belong to $H^{1}([0,1], \mathbb{R})$. System $(5)-(8)$ corresponds to a balance law system [3] actuated through one boundary.

2) Operator formulation and well-posedness: In order to motivate the definition of a weak solution for the Cauchy problem (5)-(6) with the initial condition $\left(u_{0}, v_{0}\right)$, we multiply (5)-(6) by smooth test functions $(\varphi, \psi)$ and integrate by parts. This leads to the following:

Definition 1: Let us consider a time $T>0, V \in$ $L^{2}([0, T])$. We say that $(u, v)$ is a (weak) solution to (5)(8) if $(u, v) \in\left(C^{0}\left([0, T] ; L^{2}(0,1)\right)\right)^{2}$ and

$$
\begin{gathered}
\int_{0}^{T} \int_{0}^{1}\left(-\varphi_{t}(t, x)-\lambda \varphi_{x}(t, x)-\lambda N_{u}(x) \varphi(t, 0)\right) u(t, x) \\
+\left(-\psi_{t}(t, x)+\mu \psi_{x}(t, x)-\lambda N_{v}(x) \varphi(t, 0)\right) v(t, x) \mathrm{d} x \mathrm{~d} t \\
+\int_{0}^{1} \varphi(T, x) u(T, x)-\varphi(0, x) u(0, x)+\psi(T, x) v(T, x) \\
\quad-\psi(0, x) v(0, x) \mathrm{d} x-\int_{0}^{T} \mu \psi(t, 1) V(t) \mathrm{d} t=0,
\end{gathered}
$$

for every $(\varphi, \psi) \in C^{1}([0, T] \times[0,1])^{2}$ such that

$$
\varphi(t, 1)=\rho \varphi(t, 0), \quad \psi(t, 0)=\frac{\lambda}{\mu} \varphi(t, 0) .
$$

We can now rewrite the system (5)-(8) in the abstract form

$$
\frac{d}{d t}\left(\begin{array}{l}
u \\
v
\end{array}\right)=A\left(\begin{array}{l}
u \\
v
\end{array}\right)+B V,
$$

where we can identify the operators $A$ and $B$ through their adjoints by taking formally the canonical scalar product 
of (11) with smooth test functions $(\varphi, \psi)$ and comparing with (9). The operator $A$ is thus defined by

$$
\begin{gathered}
A: D(A) \subset\left(L^{2}(0,1)\right)^{2} \rightarrow\left(L^{2}(0,1)\right)^{2} \\
\left(\begin{array}{l}
u \\
v
\end{array}\right) \longmapsto\left(\begin{array}{c}
-\lambda u_{x}(x) \\
\mu v_{x}(x)
\end{array}\right),
\end{gathered}
$$

with $D(A)=\left\{(u, v) \in\left(H^{1}(0,1)\right)^{2} \mid u(0)=v(0)+\rho u(1)+\right.$ $\left.\int_{0}^{1} N_{u}(y) u(y)+N_{v}(y) v(y), v(1)=0\right\}$.

The operator $A$ is well posed and densely defined. Adjusting the approach of [3, Appendix A], it is possible to show that $A$ generates a $C^{0}$-semigroup. Its adjoint $A^{*}$ is

$$
\begin{aligned}
A^{*}: D\left(A^{*}\right) & \subset\left(L^{2}(0,1)\right)^{2} \rightarrow\left(L^{2}(0,1)\right)^{2} \\
\left(\begin{array}{l}
u \\
v
\end{array}\right) & \longmapsto\left(\begin{array}{c}
\lambda u_{x}(x)+\lambda N_{u}(x) u(0) \\
-\mu v_{x}(x)+\lambda N_{v}(x) u(0)
\end{array}\right),
\end{aligned}
$$

with $D\left(A^{*}\right)=\left\{(u, v) \in\left(H^{1}(0,1)\right)^{2} \mid u(1)=\rho u(0), v(0)=\right.$ $\left.\frac{\lambda}{\mu} u(0)\right\}$. The operator $B \in \mathcal{L}\left(\mathbb{R}, D\left(A^{*}\right)^{\prime}\right)$ is defined by $<$ $B V,\left(\begin{array}{l}u \\ v\end{array}\right)>=\mu v(1) V$. Note that $B$ is well defined since $B V$ is continuous on $H^{1}(0,1)$ (due to the trace theorem and the fact that the graph norm associated to $D\left(A^{\star}\right)$ is equivalent to the $H^{1}$-norm). Its adjoint $B^{*} \in \mathcal{L}\left(D\left(A^{*}\right), \mathbb{R}\right)$ is then defined by

$$
B^{*}\left(\begin{array}{l}
u \\
v
\end{array}\right)=\mu v(1) \text {. }
$$

Following the approach of [8], we can show that $B$ is admissible and consequently that for every $V \in$ $L^{2}([0, T])$, there exists a unique solution to $(5)-(8)(u, v) \in$ $C^{0}\left([0, T] ; L^{2}(0,1)\right)$. This is omitted here due to space restriction. We will later show that the feedback law $V$ is actually continuous since it will correspond to a bounded feedback operator.

\section{B. Stability properties}

We now show that the stability of the system (5)-(8) implies the stability of system (2). We have the following

Lemma 1: Consider (5)-(8) with any feedback law $\bar{V}(t)$. If the solution of (5)-(8) exponentially converges to zero (with $L_{2}$-norm), then the solution of (2) exponentially converges to zero (with $L^{2}\left(\left[-\tau_{0}, 0\right]\right)$ norm).

Proof: Let us define $z(t)=u(t, 0)$. Applying the method of characteristics, we show that $z$ is the solution of (2), and that the $L^{2}$-exponential stability of $(u, v)$ implies the $L^{2}\left(\left[-\tau_{0}, 0\right]\right)$ exponential stability of $z$.

Due to Lemma 1, it is sufficient to design a feedback law that stabilizes (5)-(8). Note that such a feedback law will be expressed as a function of $u, v$ but can be causally expressed as a function of $z$ and past values of itself using $u(t, x)=$ $z\left(t-\tau_{0} x\right)$ and $v(t, x)=\bar{V}\left(t-\tau_{1}(1-x)\right)$. As a consequence, we have the following lemma

Lemma 2: Consider the operators $A^{*}$ verifying (13) and $B^{*}$ verifying (14). Under Assumption 1 we have,

$$
\forall s \in \mathbb{C}, \quad \operatorname{ker}\left(s-A^{*}\right) \cap \operatorname{ker}\left(B^{*}\right)=\{0\} .
$$

The proof is omitted due to space restrictions. Note that condition (15) is a controllability condition that can be found in [8]. It is also introduced by [12] in a much larger setting.

\section{Invertibility of some Fredholm integral operators}

The stabilization of the PDE system (5)-(8) will be done using a backstepping transformation of Fredholm type, since it offers more degrees of freedom than the traditionally used Volterra transforms. More precisely, we will consider an integral operator $\mathcal{T}:\left(L^{2}(0,1)\right)^{2} \rightarrow\left(L^{2}(0,1)\right)^{2}$ defined by

$$
\mathcal{T}\left(\begin{array}{l}
\alpha(x) \\
\beta(x)
\end{array}\right)=\left(\begin{array}{l}
\alpha(x) \\
\beta(x)
\end{array}\right)-\int_{0}^{1} K(x, y)\left(\begin{array}{l}
\alpha(y) \\
\beta(y)
\end{array}\right) d y,
$$

where $K$ is a bounded function defined on the unit square $\mathcal{S}=\left\{(x, y) \in[0,1]^{2}\right\}$. Unlike Volterra integral transformation, Fredholm transformations are not always invertible [26]. The following lemma (adjusted from [8, Lemma 2.2, Proposition 2.6]) guarantees their invertibility under several conditions.

Lemma 3: Consider a Fredholm integral operator $\mathcal{T}$ : $\left(L^{2}(0,1)\right)^{2} \rightarrow\left(L^{2}(0,1)\right)^{2}$ as defined by (16). Assume that

1) $\operatorname{ker}(\mathcal{T}) \subset D\left(A^{*}\right)$,

2) $\operatorname{ker}(\mathcal{T}) \subset \operatorname{ker}\left(B^{*}\right)$,

3) $\forall z \in \operatorname{ker}(\mathcal{T}), \mathcal{T} A^{*} z=0$

4) $\operatorname{ker}\left(s-A^{*}\right) \cap \operatorname{ker}\left(B^{*}\right)=\{0\}$, for every $s \in \mathbb{C}$.

Then, the operator $\mathcal{T}$ is invertible.

Proof: The proof follows the steps of [8, Lemma 2.2, Proposition 2.6], and can be directly derived from [24].

\section{Design of A STABILIZING CONTROL LAW}

In this section, we use a Fredholm integral operator of form (16) to map the initial hyperbolic system to an exponentially stable target system. First, we define the candidate target system. Then, we give the equations satisfied by its kernels. Finally, proving the invertibility of the Fredholm integral operator, we can define a full-state feedback control law stabilizing the initial hyperbolic system.

\section{A. Presentation of the target system}

We define a target state $(\alpha, \beta)$, that satisfies

$$
\partial_{t} \alpha+\lambda \partial_{x} \alpha=0, \quad \partial_{t} \beta-\mu \partial_{x} \beta=0,
$$

with b.c $\alpha(t, 0)=\beta(t, 0)+\rho \alpha(t, 1), \quad \beta(t, 1)=0$.

It is obviously exponentially stable in the sense of the $L_{2}$ norm. It can be rewritten using an abstract formulation as $\frac{d}{d t}\left(\begin{array}{l}\alpha \\ \beta\end{array}\right)=A_{0}\left(\begin{array}{l}\alpha \\ \beta\end{array}\right)$ where $A_{0}$ satisfies (12), and is defined on $D\left(A_{0}\right)=\left\{(\alpha, \beta) \in H^{1}(0,1)^{2} \mid \alpha(0)=\beta(0)+\right.$ $\rho \alpha(1), \beta(1)=0\}$. Its adjoint $A_{0}^{*}$ is defined by

$$
\begin{gathered}
A_{0}^{*}: D\left(A^{*}\right) \subset\left(L^{2}(0,1)\right)^{2} \rightarrow\left(L^{2}(0,1)\right)^{2} \\
\left(\begin{array}{l}
\alpha \\
\beta
\end{array}\right) \longmapsto\left(\begin{array}{c}
\lambda \alpha_{x}(x) \\
-\mu \beta_{x}(x)
\end{array}\right) .
\end{gathered}
$$

\section{B. Kernel equations}

To map the original system (5)-(8) to the target system (17)-(18), we use a Fredholm integral operator $P$ of the form (16), such that $\left(\begin{array}{l}u(x) \\ v(x)\end{array}\right)=P\left(\begin{array}{l}\alpha(x) \\ \beta(x)\end{array}\right)$. The corresponding kernels are denoted $K^{i j}=(K)_{i j}$, where $(i, j) \in$ $\{1,2\}^{2}$. They are piecewise-continuous fucntions defined on $\mathcal{S}$. Deriving the transformation with respect to time and space and integrating by parts, we obtain the following kernel equations,

$$
\partial_{\xi} K^{11}(x, \xi)+\partial_{x} K^{11}(x, \xi)=0,
$$




$$
\begin{aligned}
& \mu \partial_{\xi} K^{12}(x, \xi)-\lambda \partial_{x} K^{12}(x, \xi)=0, \\
& \lambda \partial_{\xi} K^{21}(x, \xi)-\mu \partial_{x} K^{21}(x, \xi)=0, \\
& \partial_{\xi} K^{22}(x, \xi)+\partial_{x} K^{22}(x, \xi)=0,
\end{aligned}
$$

with the boundary conditions

$$
\begin{aligned}
& \lambda K^{11}(x, 0)=\mu K^{12}(x, 0), K^{11}(x, 1)=\rho K^{11}(x, 0), \\
& \mu K^{22}(x, 0)=\lambda K^{21}(x, 0), K^{21}(x, 1)=\rho K^{21}(x, 0), \\
& K^{12}(x, 1)=0, \quad K^{22}(x, 1)=0 .
\end{aligned}
$$

The two boundary conditions (26) are necessary to guarantee the invertibility of $P$. Evaluating (16) in $x=0$, we also have

$$
\begin{aligned}
N_{u}(\xi) & -\int_{0}^{1} N_{u}(\nu) K^{11}(\nu, \xi)+N_{v}(\nu) K^{21}(\nu, \xi) \mathrm{d} \nu \\
& =-K^{11}(0, \xi)+K^{21}(0, \xi)+\rho K^{11}(1, \xi), \\
N_{v}(\xi) & -\int_{0}^{1} N_{u}(\nu) K^{12}(\nu, \xi)+N_{v}(\nu) K^{22}(\nu, \xi) \mathrm{d} \nu \\
& =-K^{12}(0, \xi)+K^{22}(0, \xi)+\rho K^{12}(1, \xi) .
\end{aligned}
$$

Theorem 1: The set of equations (20)-(26) admits a unique bounded solution on the unit square $\mathcal{S}$.

Since the kernels are defined by a complex set of equations (b.c (27)-(28)) we cannot apply classical methods [11] to prove the well-posedness. The proof of Theorem 1 requires technical computations, and is deferred to Section V.

\section{Invertibility of the Fredholm transform}

We now show the invertibility of the Fredholm integral backstepping transform $P$. We have the following theorem.

Theorem 2: Consider the Fredholm integral operator $P$ of the form (16), whose kernels are defined by (20)-(26). Then $P$ is invertible.

Proof: The adjoint operator $P^{*}$ associated to $P$, is also of the form (16). We have

$$
P^{*}\left(\left(\begin{array}{l}
\alpha(x) \\
\beta(x)
\end{array}\right)\right)=\left(\begin{array}{l}
\alpha(x) \\
\beta(x)
\end{array}\right)-\int_{0}^{1} K^{T}(y, x)\left(\begin{array}{l}
\alpha(y) \\
\beta(y)
\end{array}\right) d y .
$$

Since $P$ maps the original system (5)-(8) to the target system (17)-(18), we have for all $z \in \operatorname{ker}\left(B^{*}\right), P^{*} A^{*} z=$ $A_{0}^{*} P^{*} z$ [8]. Direct computations show that $P^{*}$ satisfies the conditions of Lemma 3 . It is therefore invertible, and so is $P$.

Since the kernel equations are well-posed by Theorem 1, the inverse operator $P^{-1}$ associated to $P$ is uniquely defined by

$$
P^{-1}\left(\begin{array}{l}
u(x) \\
v(x)
\end{array}\right)=\left(\begin{array}{l}
u(x) \\
v(x)
\end{array}\right)+\int_{0}^{1} L(x, y)\left(\begin{array}{l}
u(y) \\
v(y)
\end{array}\right) d y,
$$

where $L$ verifies $L(x, y)=K(x, y)+\int_{0}^{1} K(x, \nu) L(\nu, y) \mathrm{d} \nu$.

\section{Stabilizing control law}

Using the inverse transform (29), evaluated in $x=1$, we can design a full-state feedback controller $\bar{V}(t)$ :

$$
\bar{V}(t)=-\int_{0}^{1} L^{21}(1, \nu) u(\nu, t)+L^{22}(1, \nu) v(\nu, t) \mathrm{d} \nu .
$$

Theorem 3: The state-feedback control law $\bar{V}(t)$ defined by (30) exponentially stabilizes the hyperbolic system (5)-(8) in the sense of the $L_{2}$-norm. Thus, the control law $V(t)=$ $\frac{1}{a_{1}} \bar{V}(t)$ exponentially stabilizes the integral delay dynamics (2) in the sense of the $D_{\tau_{0}}$-norm.

Proof: With the control law $\bar{V}(t)$ defined by (30), the hyperbolic system (5)-(8) is equivalent to the target system (17)-(18). The target system is exponentially stable in the sense of the $L_{2}$-norm, and so is the initial system (5)-(8). Lemma 1 allows us to conclude the proof.

\section{WELL-POSEDNESS OF THE KERNEL EQUATIONS}

In this section, we prove the existence of a unique solution to the kernels equations (20)-(26). We rewrite these equations as two integral equations. Using Lemma 3 , we show that the associated integral operator is invertible. This guarantees the existence and uniqueness of a solution. In what follows, we only consider the case $\lambda>\mu$. The case $\lambda \leq \mu$ can be done following the same ideas. Due to space restrictions, we only give a sketch of the different proofs.

\section{A. Kernels reduction}

The first step of our proof consists in showing that the existence and uniqueness of $K^{12}(0, \xi)$ and $K^{21}(0, \xi)$ on $[0,1]$ implies the one of all the kernels on $\mathcal{S}$. We have

Lemma 4: For all $(x, \xi) \in \mathcal{S},(i, j) \in\{1,2\}^{2}, K^{i j}(x, \xi)$ can be expressed as functions of $K^{12}(0, \cdot)$ and $K^{21}(0, \cdot)$.

Proof: Applying the method of characteristics on (20)-(23), we can express $K^{i j}(x, \xi)$ as functions on the corresponding boundary values. Using the boundary conditions (24)-(26), and iterating the procedure, we can express all the kernels as functions of $K^{12}(x, 0)$ and $K^{21}(x, 0)$. Direct computations give

$$
\begin{aligned}
K^{11}(x, \xi)= & \frac{\mu}{\lambda}\left(\mathbb{1}_{[0, \xi]}(x) \rho K^{12}(x-\xi+1,0)\right. \\
& \left.+\mathbb{1}_{[\xi, 1]}(x) K^{12}(x-\xi, 0)\right), \\
K^{22}(x, \xi)= & \frac{\lambda}{\mu} \mathbb{1}_{[\xi, 1]}(x) K^{21}(x-\xi, 0) .
\end{aligned}
$$

To deal with the remaining kernels, we need to have a closer look to their definition domain and to the corresponding characteristic lines. Define $p$ as the unique integer verifying $p \frac{\mu}{\lambda} \leq 1<(p+1) \frac{\mu}{\lambda}$. We can divide $\mathcal{S}$ into different subdomains $D_{k}, k \in \llbracket 0, p+1 \rrbracket$, as illustrated on Figure 1. More precisely, we have:

- $D_{0}=\left\{0 \leq \xi \leq 1,0 \leq x \leq \frac{\mu}{\lambda}(1-\xi)\right\}$,

- $\forall k \in \llbracket 1, p-1 \rrbracket, D_{k}=\left\{0 \leq \xi \leq 1, \frac{\mu}{\lambda}(k-\xi) \leq x<\right.$ $\left.\frac{\mu}{\lambda}(k+1-\xi)\right\}$,

- $D_{p}=\left\{0 \leq \xi \leq 1, \frac{\mu}{\lambda}(p-\xi) \leq x<\min \left(1,1-\frac{\mu}{\lambda} \xi\right)\right\}$,

- $D_{p+1}=\left\{0 \leq \bar{\xi} \leq 1, \min \left(1,1-\frac{\mu}{\lambda} \xi\right) \leq x \leq 1\right\}$.

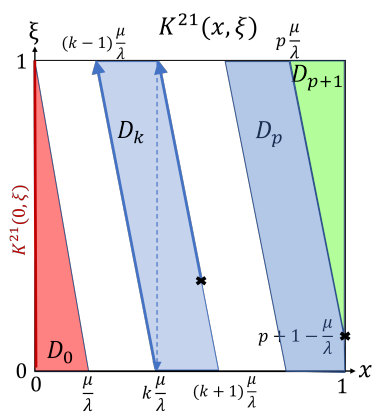

Fig. 1. Different domains in the expression of $K^{21}$ 
Using the fact that $K^{21}(x, 1)=\rho K^{21}(x, 0)$ and integrating along the characteristic lines, we obtain by iteration $\forall(x, \xi) \in$ $D_{k, k \leq p+1}, \quad K^{21}(x, \xi)=\rho^{k} K^{21}\left(0, \xi-k+\frac{\lambda}{\mu} x\right)$.

Similarly, we have

$K^{12}(x, \xi)=\mathbb{1}_{\left[0, \frac{\lambda}{\mu}(1-\xi)\right]}(x) \mathbb{1}_{[0,1]}(x) K^{12}\left(0, \xi+\frac{\mu}{\lambda} x\right)$.

This concludes the proof.

\section{B. Integral formulation}

We can now rewrite (27)-(28) as two integral equations. Henceforth, the existence and uniqueness of $K^{12}(0, \xi)$ and $K^{21}(0, \xi)$ will guarantee the well-posedness of the kernel equations according to Lemma 4. Notice first that $K^{11}(0, \xi)-\rho K^{11}(1, \xi)=0$. Using the expressions of the different kernels obtain in Section V-A, we have

$$
\begin{aligned}
K^{21}(0, \xi)= & N_{u}(\xi)-\int_{0}^{1} I_{11}(\xi, \nu) K^{12}(0, \nu) \\
& +I_{12}(\xi, \nu) K^{21}(0, \nu) \mathrm{d} \nu \\
K^{12}(0, \xi)= & -N_{v}(\xi)+\mathbb{1}_{\left[0,1-\frac{\mu}{\lambda}\right]}(\xi) \rho K^{12}\left(0, \xi+\frac{\mu}{\lambda}\right) \\
+ & \int_{0}^{1} I_{21}(\xi, \nu) K^{12}(0, \nu)+I_{22}(\xi, \nu) K^{21}(0, \nu) \mathrm{d} \nu
\end{aligned}
$$

where

$$
\begin{aligned}
& I_{11}(\xi, \nu)=\mathbb{1}_{\left[0, \frac{\mu}{\lambda}(1-\xi)\right]}(\nu) N_{u}\left(\xi+\frac{\lambda}{\mu} \nu\right) \\
&+ \mathbb{1}_{\left[\frac{\mu}{\lambda}(1-\xi), \frac{\mu}{\lambda}\right]}(\nu) \rho N_{u}\left(\xi-1+\frac{\lambda}{\mu} \nu\right) \\
& I_{12}(\xi, \nu)=\frac{\mu}{\lambda}\left[\mathbb{1}_{[\xi, 1]}(\nu) N_{v}\left(\frac{\mu}{\lambda}(\nu-\xi)\right)\right. \\
&+ \sum_{k=1}^{p} \mathbb{1}_{\left[0, \frac{\lambda}{\mu}-k+\xi\right]}(\nu) \rho^{k} N_{v}\left(\frac{\mu}{\lambda}(\nu-\xi+k)\right) \\
&\left.+\mathbb{1}_{\left[p+1+\frac{\mu}{\lambda}, 1\right]}(\xi) \mathbb{1}_{\left[0, \frac{\lambda}{\mu}-(p+1)+\xi\right]}(\nu) \rho^{p+1} N_{v}\left(\frac{\mu}{\lambda}(\nu-\xi+p+1)\right)\right] \\
& I_{21}(\xi, \nu)=\mathbb{1}_{\left[\xi, \xi+\frac{\mu}{\lambda}\right]}(\nu) \mathbb{1}_{[0,1]}(\nu) \frac{\lambda}{\mu} N_{u}\left(\frac{\lambda}{\mu}(\nu-\xi)\right) \\
& I_{22}(\xi, \nu)=\sum_{k=0}^{p} \mathbb{1}_{\left[0, \frac{\lambda}{\mu}(1-\xi)-k\right]}(\nu) \rho^{k} N_{v}\left(\xi+\frac{\mu}{\lambda}(\nu+k)\right)
\end{aligned}
$$

The expressions of $I_{i j}$ are obtained using Fubini's theorem, and the computations are quite technical. Due to space restrictions, we could not give the intermediate steps.

\section{Operator formulation}

1) Change of variable: We aim to rewrite (33)-(34) using an integral operator of form (16). To do so, we need to get rid of the term $\mathbb{1}_{\left[0,1-\frac{\mu}{\lambda}\right]}(\xi) \rho K^{12}\left(0, \xi+\frac{\mu}{\lambda}\right)$ in (34). Let $y$ be a bounded function, and define $\bar{y}$, s.t $\forall \xi \in[0,1]$ we have

$$
\tilde{y}(\xi)=y(\xi)-\mathbb{1}_{\left[0,1-\frac{\mu}{\lambda}\right]}(\xi) \rho y\left(\xi+\frac{\mu}{\lambda}\right) .
$$

This yields the following lemma

Lemma 5: The operator ? defined by (39) is invertible. More precisely, we have

$$
y(\xi)=\sum_{k=0}^{p} \rho^{k} \mathbb{1}_{\left[0,1-k \frac{\mu}{\lambda}\right]}(\xi) \tilde{y}\left(\xi+k \frac{\mu}{\lambda}\right)
$$

Using this change of variables, we can rewrite (34)-(33) as

$$
\begin{aligned}
K^{21}(0, \xi)= & N_{u}(\xi)-\int_{0}^{1} \tilde{I}_{11}(\xi, \nu) \tilde{K}^{12}(0, \nu) \\
& +I_{12}(\xi, \nu) K^{21}(0, \nu) \mathrm{d} \nu
\end{aligned}
$$

$$
\begin{aligned}
\tilde{K}^{12}(0, \xi)=- & N_{v}(\xi)+\int_{0}^{1} \tilde{I}_{21}(\xi, \nu) \tilde{K}^{12}(0, \nu) \\
& +I_{22}(\xi, \nu) K^{21}(0, \nu) \mathrm{d} \nu
\end{aligned}
$$

with $\tilde{I}_{j 1}, j \in\{1,2\}$ defined by

$$
\tilde{I}_{j 1}(\xi, \nu)=\sum_{k=0}^{p} \rho^{k} \mathbb{1}_{\left[\frac{\mu}{\lambda} k, 1\right]}(\nu) I_{j 1}\left(\nu-\frac{\mu}{\lambda} k\right) .
$$

To show that the integral equations (41)-(42) have a unique solution, we use an operator formalism.

2) Definition of the integral operator $Q$ : Introduce the Fredholm integral operator $Q$ acting on $L^{2}(0,1)^{2}$ by

$$
Q:\left(\begin{array}{l}
f \\
g
\end{array}\right) \longmapsto\left(\begin{array}{l}
f \\
g
\end{array}\right)-R\left(\begin{array}{l}
f \\
g
\end{array}\right)
$$

where $R$ is the integral operator defined by

$$
R\left(\begin{array}{c}
f(\xi) \\
g(\xi)
\end{array}\right)=\left(\begin{array}{l}
\int_{0}^{1}-I_{12}(\xi, \nu) f(\nu)+\tilde{I}_{11}(\xi, \nu) g(\nu) \mathrm{d} \nu \\
\int_{0}^{1}-I_{22}(\xi, \nu) f(\nu)+\tilde{I}_{21}(\xi, \nu) g(\nu) \mathrm{d} \nu
\end{array}\right),
$$

s.t (41)-(42) rewrites $Q\left(\begin{array}{c}K^{21}(0, \cdot) \\ -\tilde{K}^{12}(0, \cdot)\end{array}\right)=\left(\begin{array}{c}N_{u} \\ N_{v}\end{array}\right)$. Thus, if we prove that $Q$ is invertible, we will get that (41)-(42) admits a unique solution.

3) Invertibility of operator $Q$ :

Lemma 6: The operator $Q$ defined by equations (44)-(45) is invertible.

Proof: The proof is done by showing that assumptions 1) to 4 ) of Lemma 3 are verified for the operator $Q$. Indeed, due to the regularity of the kernels, $Q$ can be seen as an operator acting on the space $L^{2}(0,1)^{2}$. Assumption 4) derives from the spectral controllability of the system, and was proven in Lemma 2. Assumptions 1),2) are proved by evaluating the four coupling terms in $\xi=0$ and $\xi=1$. To prove that $\operatorname{ker}(Q)$ is stable by $A^{*}$ (Assumption 3 ), we compute the derivative of functions $(f, g) \in \operatorname{ker}(Q)$ on one side, and we integrate by parts in the integral terms on the other side. The complete proof is technical as we need to be careful to correctly define the integration domains due to the presence of characteristic functions in the integral terms. It is omitted here due to space constraints. Since $N_{u}$ and $N_{v}$ are piecewise-continuous, so are $K^{21}(0, \cdot)$ and $\tilde{K}^{12}(0, \cdot)$.

\section{Conclusion: well-posedness of the kernel equations}

We have all elements to give the proof of Theorem 1 . Proof: The invertibility of operator $Q$ given by Lemma 6 implies the existence and uniqueness of $\tilde{K}^{12}(0, \xi), K^{21}(0, \xi)$. Their regularity is a consequence of the regularity of the functions $N_{u}$ and $N_{v}$. Using Lemma 5 and (40), we have access to the boundary values $K^{12}(0, \xi)$ for all $\xi \in[0,1]$. According to Lemma 4, the four kernels $K^{i j}$ are then uniquely defined on $\mathcal{S}$. This concludes the proof.

\section{Vi. Simulation Results}

In this section, we give some simulation results to illustrate our approach. Consider the hyperbolic system (1), with coefficients $\tau_{0}=0.5, \tau_{1}=1, a_{0}=0.7<1, a_{1}=1$, and constant integral coupling terms $f_{0}=0.8, f_{1}=-0.35$. The control strategy is implemented using Matlab. We simulated our system on a time scale of $5 \mathrm{~s}$, with 51 space-discretization points in $[0,1]$. The initial condition is a constant functions $\phi^{0}=1$. We choose to work on the equivalent PDE system, 
since analyzing the $L^{2}$-norm of this system will show the $D_{\tau_{0}}$-stability of $z$ but also the convergence to zero of the control law (which is crucial for implementation and which would not be guaranteed if the actuator dynamics were directly compensated in (1)). First, the kernels $K^{i j}$ and $L^{i j}$ are computed offline using the fixed-point algorithm (successive approximation technique). The integral terms are approximated using the trapezoidal method. Then, the evolution of the hyperbolic system (5)-(8) is computed using a Godounov Scheme [17]. The control effort is computed at each time step using (30). In Figure 2, we pictured the

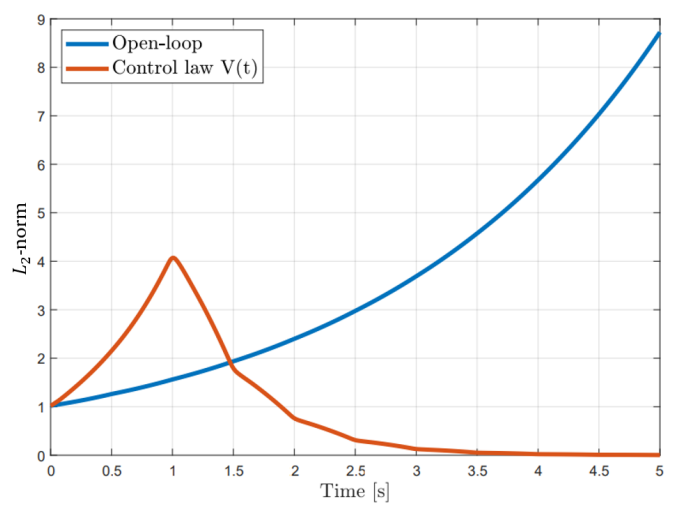

Fig. 2. Evolution of the $L_{2}$-norm of system (5)-(8).

evolution of the $L^{2}$-norm of system (5)-(8), in open-loop and closed-loop. As illustrated by the blue curve, the coefficient values were chosen such that the system is unstable in open loop. As expected, in presence of the control law (30), the system becomes exponentially stable (red curve).

\section{CONCLUDING REMARKS}

In this paper, we proposed a new approach to stabilize a general class of integral delay equations, under a spectral controllability condition. In the proposed methodology, we have first rewritten the delay equation as a scalar hyperbolic system that shares the same stability properties (comparison system). We then used the backstepping approach to map this PDE system to a simple (exponentially stable) target system. However, the configuration considered in this paper required a Fredholm transform, which is not always invertible (contrary to Volterra transforms ordinarily used). We proved the invertibility of the Fredholm transform using an operator framework inspired by [8]. The well-posedness of the kernels defining the Fredholm transform was proved using similar tools. It appeared that invertibility was a consequence of the assumed spectral controllability condition. Finally, some simulations illustrated the relevance of this approach to stabilize the integral delay dynamics. The proposed approach could be extended to the stabilization of underactuated PDE systems [1] and underactuated networks of interconnected PDE systems with actuation only available at the in-between boundary. However, the proposed strategy could not be used for industrial applications, since control law (30) requires the knowledge of all the state. We believe that an observer could be design for such systems using a similar approaches. More generally, this paper paves the way for future contributions on networks with actuation inside the graph structure. We believe that this approach could be combined with [25] to tackle a wider diversity of physical systems with an arbitrary number of PDEs or ODEs. In future contributions, we will try to extend our results to non-scalar systems. This will lead us to a general methodology for stabilizing integral delay dynamics and underactuated hyperbolic systems.

\section{REFERENCES}

[1] J. Auriol, F. Bribiesca-Argomedo, and D. Bresch-Pietri. Backstepping stabilization of an underactuated 1+2 linear hyperbolic system with a proper control. Conference on Decision and Control, 2020.

[2] J. Auriol and F. Di Meglio. An explicit mapping from linear first order hyperbolic PDEs to difference systems. Systems \& Control Letter, 2018 (accepted for publication).

[3] G. Bastin and J.-M. Coron. Stability and boundary stabilization of 1-D hyperbolic systems. Springer, 2016.

[4] N. Bekiaris-Liberis and M. Krstic. Stability of predictor-based feedback for nonlinear systems with distributed input delay. Automatica, 70:195-203, 2016.

[5] R. E. Bellman and K. L. Cooke. Differential-difference equations. 1963.

[6] F. Bribiesca-Argomedo and M. Krstic. Backstepping-forwarding control and observation for hyperbolic pdes with fredholm integrals. Automatic Control, IEEE Transactions on, 60(8):2145-2160, 2015.

[7] K. Cooke and J. Kaplan. A periodicity threshold theorem for epidemics and population growth. Mathematical Biosciences, 31(1):87-104, 1976.

[8] J.-M. Coron, L. Hu, and G. Olive. Stabilization and controllability of first-order integro-differential hyperbolic equations. Journal of Functional Analysis, 271(12):3554-3587, 2016.

[9] J.-M. Coron, L. Hu, and G. Olive. Finite-time boundary stabilization of general linear hyperbolic balance laws via Fredholm backstepping transformation. Automatica, 84:95-100, 2017.

[10] M. Courtemanche, J. P. Keener, and L. Glass. A delay equation representation of pulse circulation on a ring in excitable media. SIAM Journal on Applied Mathematics, 56(1):119-142, 1996.

[11] F. Di Meglio, F. B. Argomedo, L. Hu, and M. Krstic. Stabilization of coupled linear heterodirectional hyperbolic pde-ode systems. Automatica, 87:281-289, 2018.

[12] H. Fattorini. Some remarks on complete controllability. SIAM Journal on Control, 4(4):686-694, 1966.

[13] J. Hale and S.M. Verduyn Lunel. Strong stabilization of neutral functional differential equations. IMA Journal of Mathematical Control and Information, 19(1 and 2):5-23, 2002.

[14] J.K. Hale and S.M. Verduyn Lunel. Introduction to functional differential equations. Springer-Verlag, 1993.

[15] L. Hu, F. Di Meglio, R. Vazquez, and M. Krstic. Control of homodirectional and general heterodirectional linear coupled hyperbolic PDEs. IEEE Transactions on Automatic Control, 61(11):3301-3314, 2016.

[16] M. Krstic and A. Smyshlyaev. Backstepping boundary control for first-order hyperbolic PDEs and application to systems with actuator and sensor delays. Systems \& Control Letters, 57(9):750-758, 2008.

[17] R. J. LeVeque. Finite volume methods for hyperbolic problems. Cambridge university press, 2002.

[18] H. Logemann, R. Rebarber, and G. Weiss. Conditions for robustness and nonrobustness of the stability of feedback systems with respect to small delays in the feedback loop. SIAM Journal on Control and Optimization, 34(2):572-600, 1996.

[19] H. Mounier. Algebraic interpretations of the spectral controllability of a linear delay system. In Forum Mathematicum, volume 10, pages 39-58. De Gruyter, 1998.

[20] S.-I. Niculescu. Delay effects on stability: a robust control approach, volume 269. Springer Science \& Business Media, 2001.

[21] S.-I. Niculescu. On delay-dependent stability under model transformations of some neutral linear systems. International Journal of Control, 74(6):609-617, 2001.

[22] L. Pandolfi. Stabilization of neutral functional differential equations. Journal of Optimization Theory and Applications, 20(2):191-204, 1976.

[23] A. Ponomarev. Reduction-based robustness analysis of linear predictor feedback for distributed input delays. IEEE Transactions on Automatic Control, 61(2):468-472, 2015.

[24] J. Redaud, J. Auriol, and S. I. Niculescu. Observer design for a class of delay systems using a fredholm transform. 16th IFAC Workshop on Time Delay Systems, 2021.

[25] J. Redaud, J. Auriol, and S. I. Niculescu. Output-feedback control of an underactuated network of interconnected hyperbolic pde-ode systems. System and control letters, under revision.

[26] K. Yoshida. Lectures on differential and integral equations, volume 10 Interscience Publishers, 1960. 